\title{
An application of contact melting theory to skates sliding on ice
}

\author{
P. J. Montgomery \\ Mathematics Program, University of Northern British Columbia, \\ 3333 University Way, Prince George, B.C., Canada
}

\begin{abstract}
The problem of a skate blade sliding over ice is a complex and classic problem, with an early form considered by Reynolds over a century ago. The problem is revisited herein: a thin layer of water in between the skate blade and the ice surface is assumed to exist, and acts as a lubricant for the sliding motion of the skate blade. The existence of the melt layer is caused by viscous friction in the liquid film itself, instead of pressure melting. Governing equations are considered for a Newtonian and inviscid fluid of constant density. These equations are reduced by considering some scaling analysis to determine the negligible terms, and a simpler planar flow is considered. Through some straightforward manipulations of the governing equations, the viscous stress on the surface of the skate blade is analytically expressed as a function of the depth of the melt layer. Other results are used to posit an approximate expression for the non-constant depth of the melt layer, and this is used to calculate the frictional force. The results are compared to others in the area, and limitations on the modelling are discussed.
\end{abstract}

Keywords: multiphase flow, fluid dynamics, stefan problem.

\section{Introduction}

Contact melting is an area of research in which two solids interact in such a way that one of the solids partially melts at the surface to create a thin melted layer between the two solids which acts to lubricate the motion between the two parts. A relatively recent review of the industrial and engineering applications of this theory has been completed by Bejan [1], although the area of focus of this manuscript is on the application of the contact melting theory to describe a skate blade sliding over ice. The classical mechanism of melting due to surface pressure has been 


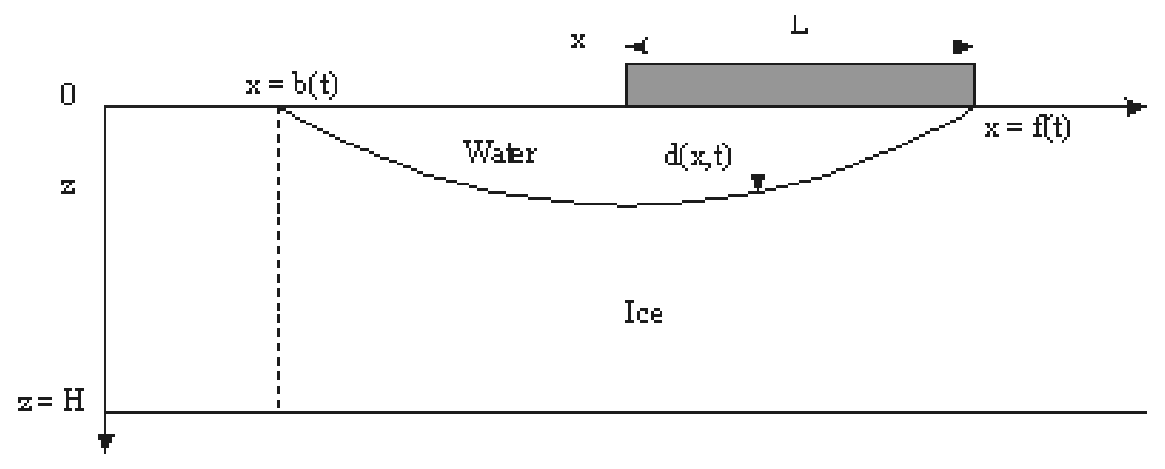

Figure 1: Geometry of the skate blade variables (not to scale).

shown to be essentially incorrect, and the melted layer is considered to be created due to the viscous friction of the motion (see [2] and the references therein.)

In a previous study, the concept of a thin lubricating layer of water was used to permit a study of the frictional drag on an object sliding over ice [3]. In Summers and Montgomery [3] the two main limitations on the theory were an assumption that the horizontal pressure gradient was negligible, and the depth of the melt layer was undetermined. In a more recent work Penny et al [4] posed a partial solution for the depth of the melt layer in the form of a nonlinear nonhomogeneous first order ordinary differential equation, thus partially removing one of the previous limitations.

In this study, the theory of contact melting is applied to determine the frictional force on a skate blade over ice. The theory follows that of Fowler and Bejan [5] and implements the results of Penny et al [4] to create an expression for the viscous drag force on the skate blade. In Section 2, the governing equations for the melt layer are derived fairly thoroughly as this seems to be something that is lacking in the standard literature. In two subsections, the equations are simplified by assuming a small aspect ration (vertical to horizontal) and a thin model layer is developed. The equations are used in Section 3 to create a solution for the pressure and velocity in the melt layer without any assumptions on the depth of the layer. By using an approximate solution similar to the numerical results of Penny et al [4] an expression for the viscous drag force is derived which is similar to previous results [6].

\section{Model equations}

A sketch of the initial skate blade geometry is depicted in Figure 1, where a solid metal blade of length $L$ overlies a thin layer of liquid melted from the ice beneath. It is assumed that the start of the melt layer occurs coincidentally with the beginning of the skate blade, and that there are no asperities in the ice which would cause uneven contact [5]. 
To model the liquid layer, the fluid is assumed to be Newtonian, and therefore satisfies the Navier-Stokes equations [7] in a Cartesian coordinate frame of reference;

$$
\frac{1}{\rho} \frac{D \rho}{D t}+\nabla \cdot \vec{u}=0
$$

for mass conservation, and the momentum equation

$$
\rho \frac{D \vec{u}}{D t}=-\nabla p+\rho \vec{g}+\mu\left[\nabla^{2} \vec{u}+\frac{1}{3} \nabla(\nabla \cdot \vec{u})\right] .
$$

In equations (1) and (2), $\rho$ and $\vec{u}=(u, v, w)$ denote the fluid density and velocity, $p$ the pressure, $\mu$ the viscosity, and $\vec{g}$ the gravitational force per unit mass. The standard material derivative notation $\frac{D}{D t}=\frac{\partial}{\partial t}+\vec{u} \cdot \nabla$ is also used. The viscosity is assumed to be a constant as the temperature of the liquid layer is expected to be very near the freezing point for the duration of the fluid motion.

To complete the description of the fluid and close the governing equations, a thermal energy equation is required from the first law of thermodynamics. If $e$ denotes the specific internal energy, $\vec{q}$ the outward heat flux vector per unit area, and there is no internal heat generation, then energy conservation can be written in the form of a thermal energy equation (heat equation,) [7, 6]

$$
\rho \frac{D e}{D t}=-\nabla \cdot \vec{q}-p(\nabla \cdot u)+\mu \phi .
$$

The viscous dissipation function, $\phi$, in equation (3) can be written in its full form as [6]

$$
\begin{aligned}
\phi= & 2\left[\left(\frac{\partial u}{\partial x}\right)^{2}+\left(\frac{\partial v}{\partial y}\right)^{2}+\left(\frac{\partial w}{\partial z}\right)^{2}\right]-\frac{2}{3}\left[\frac{\partial u}{\partial x}+\frac{\partial v}{\partial y}+\frac{\partial w}{\partial z}\right]^{2} \\
& +\left[\left(\frac{\partial u}{\partial y}+\frac{\partial v}{\partial x}\right)^{2}+\left(\frac{\partial v}{\partial z}+\frac{\partial w}{\partial y}\right)^{2}+\left(\frac{\partial w}{\partial x}+\frac{\partial u}{\partial z}\right)^{2}\right]
\end{aligned}
$$

Equations (1) to (4) are simplified in the next subsection and adapted to the geometry of Figure 1.

\subsection{Incompressibility and symmetry}

To simplify the governing equations (1)-(4), some assumptions are of course necessary. Fortunately, these are well justified, and the resultant equations can be seen to capture the essential components of the model without making unjustified assumptions merely for the sake of simplicity. The first simplification arises from specifying a few standard properties of the fluid layer, and it is thus assumed that the fluid is of constant density and incompressible. The second assumption is to consider only planar motion in the horizontal and vertical plane, neglecting any variation across the skate blade (i.e. the $y$ direction). Although the assumption of incompressibility is standard $[6,5]$ the assumption of a uniformly placed 
skate blade is restrictive as often blades are subject to varying forces during an actual skating stroke [4]. However, in order to obtain an advancement of the theory, the variation of forces within the skate blade are assumed to average out, and the assumptions thus permit equation (1) to be written as the standard assumption of incompressibility in two spatial dimensions [7]

$$
\frac{\partial u}{\partial x}+\frac{\partial w}{\partial z}=0
$$

The restriction to planar motion simplifies the vector equation (2) by one component, and only the $x$ and $z$ components remain. Substitution of equation (5) into equation (2) gives these two components as

$$
\rho \frac{D u}{D t}=-\frac{\partial p}{\partial x}+\mu\left(\frac{\partial^{2} u}{\partial x^{2}}+\frac{\partial^{2} u}{\partial z^{2}}\right),
$$

and

$$
\rho \frac{D w}{D t}=-\frac{\partial p}{\partial z}-\rho g+\mu\left(\frac{\partial^{2} w}{\partial x^{2}}+\frac{\partial^{2} w}{\partial z^{2}}\right),
$$

where the gravitational force is taken to be in the vertical direction, and the material derivative simplifies to $\frac{D}{D t}=\frac{\partial}{\partial t}+u \frac{\partial}{\partial x}+w \frac{\partial}{\partial z}$.

The thermal energy equation (3) is rewritten by using the linear relation of internal energy $e$ to temperature $T, e=c_{w} T$ where $c_{w}$ is the specific heat capacity of water [6]. In addition, the constitutive relation given by Fourier's law of heat conduction [7],

$$
\vec{q}=-k_{w} \nabla T
$$

is used where $k_{w}$ is the thermal conductivity of water. These changes, together with the incompressibility assumption (5) give

$$
\rho c_{w} \frac{D T}{D t}=k_{w}\left(\frac{\partial^{2} T}{\partial x^{2}}+\frac{\partial^{2} T}{\partial z^{2}}\right)+\mu \phi,
$$

where $\phi$ is simplified from (4) to

$$
\phi=2\left[\left(\frac{\partial u}{\partial x}\right)^{2}+\left(\frac{\partial w}{\partial z}\right)^{2}\right]+\left(\frac{\partial w}{\partial x}+\frac{\partial u}{\partial z}\right)^{2}
$$

In the next subsection, the governing equations are further simplified to account for the specific geometry of thin layer flow.

\subsection{Small aspect ratio}

The second major simplification applied to the governing equations is to neglect those terms which may be extremely small compared to the other terms. To that 
end nondimensional variables denoted with a tilde $(\tilde{)}$ are introduced by factoring out dimensional constants according to

$$
(u, w)=(U \tilde{u}, W \tilde{w}),(x, z)=(X \tilde{x}, Z \tilde{z}), t=\frac{X}{U} \tilde{t}, T=\Lambda \tilde{T}, p=-\rho g z+P \tilde{p} .
$$

Substitution of the new variables (11) into the incompressibility equation (5) gives

$$
\frac{U}{X} \frac{\partial \tilde{u}}{\partial \tilde{x}}+\frac{W}{Z} \frac{\partial \tilde{w}}{\partial \tilde{z}}=0
$$

from which it is inferred that $W=\delta U$, where $\delta=Z / X$ is the aspect ratio of vertical to horizontal scales.

The momentum equations (6) and (7) are written in expanded form with the new variables (11) and use of the previous scaling notation as

$$
\rho \frac{U^{2}}{X}\left(\frac{\partial \tilde{u}}{\partial \tilde{t}}+\tilde{u} \frac{\partial \tilde{u}}{\partial \tilde{x}}+\tilde{w} \frac{\partial \tilde{u}}{\partial \tilde{z}}\right)=-\frac{P}{X} \frac{\partial \tilde{p}}{\partial \tilde{x}}+\mu \frac{U}{\delta^{2} X^{2}}\left(\delta^{2} \frac{\partial^{2} \tilde{u}}{\partial \tilde{x}^{2}}+\frac{\partial^{2} \tilde{u}}{\partial \tilde{z}^{2}}\right),
$$

and

$$
\rho \frac{\delta U^{2}}{X}\left(\frac{\partial \tilde{w}}{\partial \tilde{t}}+\tilde{u} \frac{\partial \tilde{w}}{\partial \tilde{x}}+\tilde{w} \frac{\partial \tilde{w}}{\partial \tilde{z}}\right)=-\frac{P}{\delta X} \frac{\partial \tilde{p}}{\partial \tilde{z}}+\mu \frac{U}{\delta X^{2}}\left(\delta^{2} \frac{\partial^{2} \tilde{w}}{\partial \tilde{x}^{2}}+\frac{\partial^{2} \tilde{w}}{\partial \tilde{z}^{2}}\right) .
$$

Similarly, equations (9) and (10) become

$$
\rho c_{w} \frac{\Lambda U}{X}\left(\frac{\partial \tilde{T}}{\partial \tilde{\tau}}+\tilde{u} \frac{\partial \tilde{T}}{\partial \tilde{x}}+\tilde{w} \frac{\partial \tilde{T}}{\partial \tilde{z}}\right)=k_{w} \frac{\Lambda}{\delta^{2} X^{2}}\left(\delta^{2} \frac{\partial^{2} \tilde{T}}{\partial \tilde{x}^{2}}+\frac{\partial^{2} \tilde{T}}{\partial \tilde{z}^{2}}\right)+\mu \phi,
$$

and

$$
\phi=\frac{U^{2}}{\delta^{2} X^{2}}\left[2 \delta^{2}\left(\frac{\partial \tilde{u}}{\partial \tilde{x}}\right)^{2}+2 \delta^{2}\left(\frac{\partial \tilde{w}}{\partial \tilde{z}}\right)^{2}+\left(\delta^{2} \frac{\partial \tilde{w}}{\partial \tilde{x}}+\frac{\partial \tilde{u}}{\partial \tilde{z}}\right)^{2}\right] .
$$

For a very thin layer, the aspect ratio is necessarily small, perhaps of the order of $10^{-6}$ [4]. If the terms which are of order $\delta^{2}$ are neglected from equations (13) to (16) the resulting approximate equations are drastically reduced, and result in a problem similar to Couette flow. For the skate blade problem, the pressure scaling term $P$ is unknown, but is expected to be relatively large and therefore all terms which are $O\left(\delta^{2}\right)$ are neglected except those which contain the scaling $P$. The resulting equations are stated as three results (since equations (15) and (16) are combined):

$$
0=-\frac{P \delta^{2}}{X} \frac{\partial \tilde{p}}{\partial \tilde{x}}+\mu \frac{U}{X^{2}} \frac{\partial^{2} \tilde{u}}{\partial \tilde{z}^{2}}, \quad 0=-\frac{P}{X} \frac{\partial \tilde{p}}{\partial \tilde{z}}+\mu \frac{U}{X^{2}} \frac{\partial^{2} \tilde{w}}{\partial \tilde{z}^{2}},
$$

and

$$
0=k_{w} \frac{\Lambda}{X^{2}} \frac{\partial^{2} \tilde{T}}{\partial \tilde{z}^{2}}+\mu \frac{U^{2}}{X^{2}}\left(\frac{\partial \tilde{u}}{\partial \tilde{z}}\right)^{2} .
$$

By using equation (17) scalings can be compared to finally deduce that the pressure scaling can be fixed as $P=\mu \frac{U}{\delta^{2} X}$, which then justifies the assumption of 
a large pressure scaling and yields the approximate equations, now stated dimensionally (and neglecting the vertical hydrostatic part of the pressure term) as

$$
\begin{gathered}
0=-\frac{\partial p}{\partial x}+\mu \frac{\partial^{2} u}{\partial z^{2}}, \\
0=-\frac{\partial p}{\partial z}
\end{gathered}
$$

and

$$
0=k_{w} \frac{\partial^{2} T}{\partial z^{2}}+\mu\left(\frac{\partial u}{\partial z}\right)^{2} .
$$

Equations (19), (20), and (21) are an extension of those used previously [3] as the temperature variation is included. They have been used previously [5] but with minimal justification as to their derivation, and it was felt by the author that a thorough exposition was warranted.

\section{Thin layer solution}

The approximate equations derived in the previous section are employed to give a first solution for the skate blade problem following the method of Fowler and Bejan [5]. An inertial frame of reference with the blade of the skate, and oriented such that the layer is of positive height in positive horizontal position is used (essentially a rotation of the geometry in Figure 1. As such, the steady state problem moving with the speed of the skate is considered, and transition effects to this steady state solution are not obtained. First, using equation (20) we note that $p=p(x)$ only, and therefore, equation (19) may be integrated over the vertical domain $0 \leq z \leq h$. Using the boundary conditions $u(x, 0)=0$ and $u(x, h)=V$ the double integration of equation (19) yields the solution

$$
u(x, z)=\frac{1}{2 \mu} \frac{d p}{d x}(z-h) z+\frac{V}{h} z .
$$

The solution (22) is not completely specified as the horizontal pressure gradient is still unknown. However, the incompressibility equation (5) may be used by substituting the expression (22). The result is

$$
\frac{1}{2 \mu}\left[\frac{d^{2} p}{d x^{2}}(z-h) z-\frac{d p}{d x} \frac{d h}{d x} z\right]-\frac{V}{h^{2}} \frac{d h}{d x} z+\frac{\partial w}{\partial z}=0 .
$$

Integrating equation (23) vertically and using the kinematic boundary condition $w=0$ at $z=0$ gives

$$
\frac{1}{2 \mu}\left[\frac{d^{2} p}{d x^{2}}\left(\frac{1}{3} z-\frac{1}{2} h\right) z^{2}-\frac{1}{2} \frac{d p}{d x} \frac{d h}{d x} z^{2}\right]-\frac{1}{2} \frac{V}{h^{2}} \frac{d h}{d x} z^{2}+w=0 .
$$


Evaluating equation (24) along the contour $z=h$ where $w=0$ is assumed gives a second order ODE for $p(x)$,

$$
\frac{1}{2 \mu}\left[\frac{d^{2} p}{d x^{2}}\left(-\frac{1}{3} h+\frac{1}{2} h\right) h^{2}+\frac{1}{2} \frac{d p}{d x} \frac{d h}{d x} h^{2}\right]-\frac{1}{2} \frac{V}{h^{2}} \frac{d h}{d x} h^{2}=0,
$$

which can be rearranged to a simpler form via the chain rule,

$$
\frac{d}{d x}\left(h^{3} \frac{d p}{d x}\right)=-6 \mu V \frac{d h}{d x} .
$$

The pressure gradient can now be expressed by integrating equation (26) and using a boundary condition at the end of the skate blade where it is assumed that the gradients vanish. This assumption yields

$$
\frac{d p}{d x}=\frac{6 \mu V\left(h_{0}-h\right)}{h^{3}}
$$

where $h_{0}$ is the maximum depth of the liquid layer.

Finally, substitution of equation (27) into equation (22) gives the solution

$$
u(x, z)=\frac{V z}{h}\left[\frac{3\left(h_{0}-h\right)(z-h)}{h^{2}}+1\right] .
$$

\section{Viscous drag on the skate blade}

The velocity of the lubricating liquid layer given by equation (28)permits the viscous drag force, $F$, on the skate blade to be calculated by integrating of the shear stress along the length of the skate

$$
F=\left.\int_{0}^{L} \mu \frac{\partial u}{\partial z}\right|_{z=0} d x
$$

Substitution of equation (28) into the above gives

$$
F=\mu V \int_{0}^{L} \frac{\left(3 h_{0}-2 h\right)}{h^{2}} d x
$$

At this point, some expression for the depth $h(x)$ of the liquid layer is needed to complete the calculation for the drag on the skate blade. A separate analysis containing a discussion of this has been completed [4], however an analytic expression for $h(x)$ is not yet available. A graph obtained from the numerical approximation of the results of [4] is given in Figure 2, along with the approximate solution ( $L=40 \mathrm{~cm}$ is used for the calculations in Figure 2)

$$
h(x)=h_{0}\left(\frac{x}{L}\right)^{\frac{1}{3}}
$$




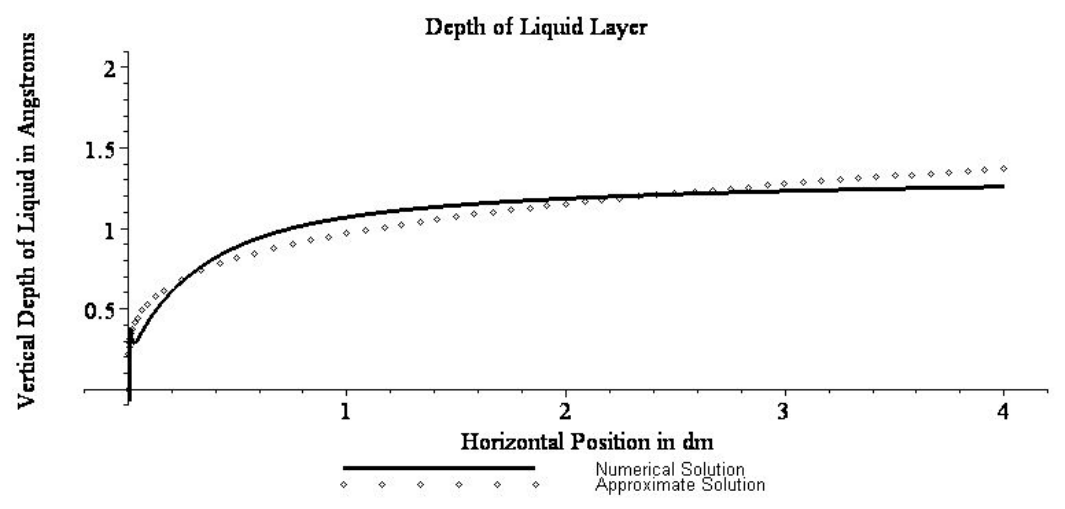

Figure 2: Numerical and approximate solutions of the depth, $h(x)$, of the liquid layer. A more complete description is given by Penny et al [4].

Substituting the assumption (31) into the integral (30) gives

$$
F=\left.\mu V \frac{3 L}{h_{0}} 3 L\left[3\left(\frac{x}{L}\right)^{\frac{1}{3}}-\left(\frac{x}{L}\right)^{\frac{2}{3}}\right]\right|_{x=0} ^{x=L}=\frac{6 \mu V L}{h_{0}}
$$

The result (32) is similar in behaviour to a result obtained by Bejan [6] except for the factor of 6 , where the layer was assumed to be of constant height, and knowledge of the depth $h(x)$ was not required. It is clear that there is a great degree of sensitivity to the expression used for the layer depth, and an approximation such as (31) will impact the tangential force heavily. Importantly, the Stefan problem [8] is not required to be solved, as long as an approximation for the depth of the melt layer is known.

\section{Summary}

The concept of a viscous lubricating melt layer was used to determine the frictional force on an idealised problem similar to that of a skate blade. By approximating the governing equations, explicit expressions for the pressure gradient and velocity were obtained as functions of the depth of the liquid layer. By using a power law to approximate the depth of the melt layer, the viscous shear stress was calculated directly to yield a result similar to previous results obtained elsewhere [6]. The results are limited by the accuracy of the assumptions and the expression used for the depth of the melt layer, which is an essential assumption. 


\section{Acknowledgements}

The author PJM gratefully acknowledges UNBC for assistance in the form of a conference travel grant, and NSERC's essential support from a discovery grant.

\section{References}

[1] Bejan, A., Contact melting heat transfer and lubrication, Adv. Heat Transfer, 24, pp. 129-155, 1994.

[2] Bejan, A., The fundamentals of sliding contact melting and friction, J. Heat Transfer, 111, pp. 13-20, 1989.

[3] Summers, A.E. \& Montgomery, P.J., Investigation of the motion of a fixed rotating solid body over ice with a thin fluid interface. Advances in Fluid Mechanics V, eds. C.A. Brebbia, A.C. Mendes \& M. Rahman, WIT Press: Ashurst, pp. 247-256, 2004.

[4] Penny, A., Lozowski, E., Forest, T., Fong, C., Maw, S., Montgomery, P. \& Sinha, N., Speedskate Ice Friction: Review and Numerical Model - FAST 1.0., Proceedings of the 11th International Conference on the Physics and Chemistry of Ice (PCI 2006), Royal Society for Chemistry, in press, 2006.

[5] Fowler, A.J. \& Bejan, A., Contact melting during sliding on ice, Int. J. Heat Mass Transfer, 36(5), pp. 1171-1179, 1993.

[6] Bejan, A. Convection Heat Transfer, $2^{\text {nd }}$ edn., John Wiley and Sons: New York, 1995.

[7] Kundu, P.K., Fluid Mechanics, Academic Press: San Diego, 1990.

[8] Crank, J.C., Free and Moving Boundary Problems, Clarendon Press: Oxford, 1984. 